\title{
Hochselektive und hochempfindliche Fasersensorik für die Umweltanalytik und Medizin
}

\author{
Torsten Frosch ${ }^{1,2}$, Di Yan ${ }^{1}$, Timea Bögözi ${ }^{1}$, Tobias Jochum ${ }^{1}$, Juergen Popp ${ }^{1,2}$ \\ ${ }^{1}$ Leibniz-Institut für Photonische Technologien, Albert-Einstein-Straße 9, 07745 Jena, Deutschland \\ ${ }^{2}$ Institut für Physikalische Chemie und Abbe Center of Photonics, Friedrich-Schiller-Universität, \\ Helmholtzweg 4, 07743 Jena, Deutschland \\ torsten.frosch@uni-jena.de
}

\begin{abstract}
Einleitung
Faserverstärkte Raman-Spektroskopie (FERS, fiber enhanced Raman spectroscopy) ist eine neuartige, vielseitige Methode für die schnelle online-Analyse biogener Gase in Multigasgemischen [13] sowie dem Nachweis von Pharmazeutika in Lösungen [4].

Konventionelle Raman-Spektroskopie bietet eine exzellente chemische Selektivität für die simultane Identifizierung verschiedener Gasen in komplexen Mischungen. Jedoch ist die Sensitivität nicht ausreichend für den Nachweis geringer Konzentrationen im ppm-Bereich. Ebenso ist es notwendig Pharmazeutika selektiv und in sehr niedrigen Konzentrationen in wässriger Lösung für die Umweltanalytik und medizinische Diagnostik nachzuweisen.
\end{abstract}

Schlagwörter: Faserverstärkte Raman Spektroskopie; biogene Gase; Pharmazeutika; Umweltanalytik; Gassensorik; Atemgasanalyse

Der Nachteil der geringen Sensitivität der Raman-Spektroskopie kann mittels der Erforschung und Entwicklung innovativer optischer Hohlfasern [5, 6] ausgeglichen werden. Diese neuartigen Hohlfasern dienen einerseits als optischer Wellenleiter für eine verlustarme Führung des Lichtes und andererseits als minimierter Analyt-Container. Somit kann eine wesentlich effizientere Wechselwirkung bei minimalem Analytbedarf erzielt werden und es wird eine erhöhte Sensitivität bis in die gewünschten Nachweisbereiche ermöglicht [3, 4].

Die Analyse biogener Gase und deren Austausch ermöglichen ein besseres Verständnis komplexer Umweltprozesse [3]. Die hochempfindliche Raman-Gassensorik eröffnet in der Umweltforschung vielfältige neuartige Möglichkeiten, wie bspw. für das Monitoring von ${ }^{13} \mathrm{CO}_{2}$-Labeling-Experimenten, der Analyse klimasensitiver Ökosysteme, der Auswirkungen von Umweltstress sowie dem Nachweis von Schadstoffen in der Luft und im Boden.

Auf dem Gebiet der nichtinvasiven Früherkennung von Krankheiten, erlaubt FERS die schnelle Analyse von gasförmigen und volatilen Krankheitsmarkern in der Atemluft [1, 2]. Beispielsweise kann mittels FERS ein Wasserstoffatemtest, d.h. der Nachweis von $\mathrm{H}_{2}$ und $\mathrm{CH}_{4}$ in den Bereichen von 10 bis 100 ppm im Atem von Patienten mit Laktoseintoleranz, durchgeführt werden [1, 2].

Die hohe Faserverstärkung kann in weiteren Schritten mit UV- und Resonanzverstärkungen kombiniert werden. Somit ist ein hochsensitiver Nachweis der Antimalariawirkstoffe Chloroquin und Mefloquin im sub- $\mu \mathrm{M}$ Bereich möglich [4].

Aufgrund der Miniaturisierbarkeit von FERSAufbauten weißt die hochselektive und hochempfindliche Raman-Fasersensorik ein hohes Potenzial für umweltanalytische Feldmessungen und die medizinische point-ofcare Diagnostik auf.

\section{Literatur}

[1] T. Boegoezi, J. Popp and T. Frosch, Bioanalysis 2015, 7, 281-284.

[2] S. Hanf, T. Boegoezi, R. Keiner, T. Frosch and J. Popp, Analytical Chemistry, 2015, 87, 982-988.

[3] S. Hanf, R. Keiner, D. Yan, J. Popp and T. Frosch, Analytical Chemistry, 2014, 86, 52785285.

[4] T. Frosch, D. Yan and J. Popp, Analytical Chemistry 2013, 85, 6264-6271.

[5] A. Hartung, J. Kobelke, A. Schwuchow, K. Wondraczek, J. Bierlich, J. Popp, T. Frosch and M. A. Schmidt, Optics express, 2015, 23, 25572565. 
[6] A. Hartung, J. Kobelke, A. Schwuchow, K. Wondraczek, J. Bierlich, J. Popp, T. Frosch and M. A. Schmidt, Optics express, 2014, 22, 1913119140.

\section{Danksagung}

Förderung durch die Deutsche Forschungsgemeinschaft (SFB 1076, AquaDiva) und das Land Thüringen unter Co-Finanzierung durch EFRE (FKZ: 2012 FGR 0013). 